\title{
TOPICAL CYCLOSPORIN A 2\% IN THE TREATMENT OF VERNAL KERATOCONJUNCTIVITIS
}

\author{
J. MENDICUTE, C. ARANZASTI, F. EDER, J. I. OSTOLAZA and M. SALABERRIA \\ San Sebastián, Spain
}

\begin{abstract}
SUMMARY
Topical cyclosporin $A$ at a $2 \%$ concentration was used for 6 months in the management of 2 patients with vernal keratoconjunctivitis who had failed to respond to conventional therapy. We used one drop every 6 hours in both eyes during the first month and every 12 hours during the remaining 5 months. Clinical controls were carried out weekly during the first month, monthly during the 6 month period and every 2 months thereafter. Cyclosporin A blood levels and serum creatinine were regularly monitored in both patients. Within the first month, both the symptoms and signs of the condition, in particular papillary proliferations, improved significantly and these results were maintained throughout the entire period of treatment and during 2 years of follow-up with conservative management (artificial tears).
\end{abstract}

Vernal keratoconjunctivitis is a chronic, recurrent ocular allergic disease that primarily affects young people and is characterised by raised conjunctival papillae on the upper tarsal plate, diffuse keratitis and epithelial erosions in moderate and severe cases, mucous discharge and occasionally the presence of Trantas' dots in patients with limbal forms.Vernal keratoconjunctivitis, although clearly seasonal during the first attacks and self-limiting in most cases, can become a chronic entity with persistent symptoms throughout the year. Mild cases can be managed by conservative measures. For more tenacious cases, the use of corticosteroid eye drops is effective. However, a few cases become refractory and symptoms are relieved only by systemic corticosteroids. Because of the possible sequelae of long-term use of corticosteroids or their failure in treatment, other pharmacologic approaches have been suggested.

Cyclosporin $\mathrm{A}$ is a potent immunoregulator with a major impact on the management of immune-

From: Hospital de Guipuzcoa, Servicio de Oftalmología, San Sebastián, Guipuzcoa, Spain.

Correspondence to: Javier Mendicute, Azara 17-1ㅇE, E-20800

Zarauz, Guipuzcoa, Spain. mediated inflammatory conditions. Cyclosporin inhibits mainly interleukin- 2 production and modulates the helper/suppressor interaction.

Topical administration of cyclosporin $\mathrm{A}$ has been demonstrated to be useful in the treatment of ocular inflammatory surface diseases that have failed conventional therapy, such as the prevention of corneal allograft rejection or high-risk keratoplasty, ligneous conjunctivitis, rheumatoid corneal ulceration including corticosteroid-dependent or corticosteroid-resistant severe vernal keratoconjunctivitis. ${ }^{1-6}$

We report 2 cases of severe vernal keratoconjunctivitis successfully treated with topical cyclosporin A for 6 months with remission, in particular of giant papillae, not documented in other clinical trials. ${ }^{3}$

\section{Case 1}

\section{CASE REPORTS}

A 9-year-old boy showed a severe chronic bilateral palpebral type of vernal keratoconjunctivitis refractory to local treatment with corticosteroid eyedrops and cromolyn solutions. The parents were instructed to discontinue all medication. At the time the topical cyclosporin was started, symptoms were present (itching, photophobia, tear-shedding, pain) and ocular examination found conjunctival hyperaemia, severe papillary proliferations and extensive corneal involvement.

\section{Case 2}

A 23-year-old man who had suffered from severe bilateral chronic vernal keratoconjunctivitis since he was 10 years old, had previously used topical corticosteroids and cromolyn sodium eyedrops without relief of symptoms (intense tear-shedding, photophobia, pain and mucous discharge) or signs (giant papillae, conjunctival hyperaemia, punctate keratitis of more than three quadrants and limbal neovascularisation at 12 o'clock) in both eyes (Figs. 1, 2). 


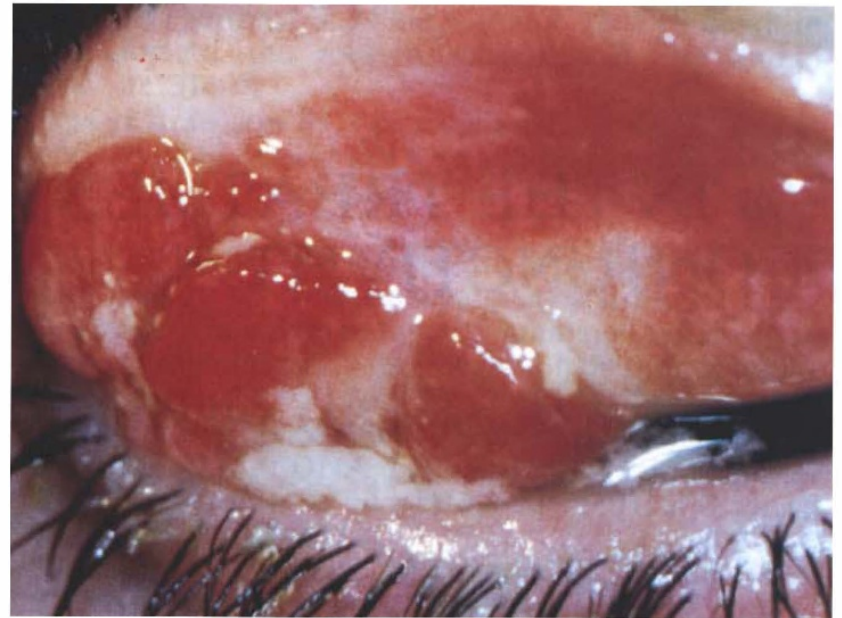

(a)

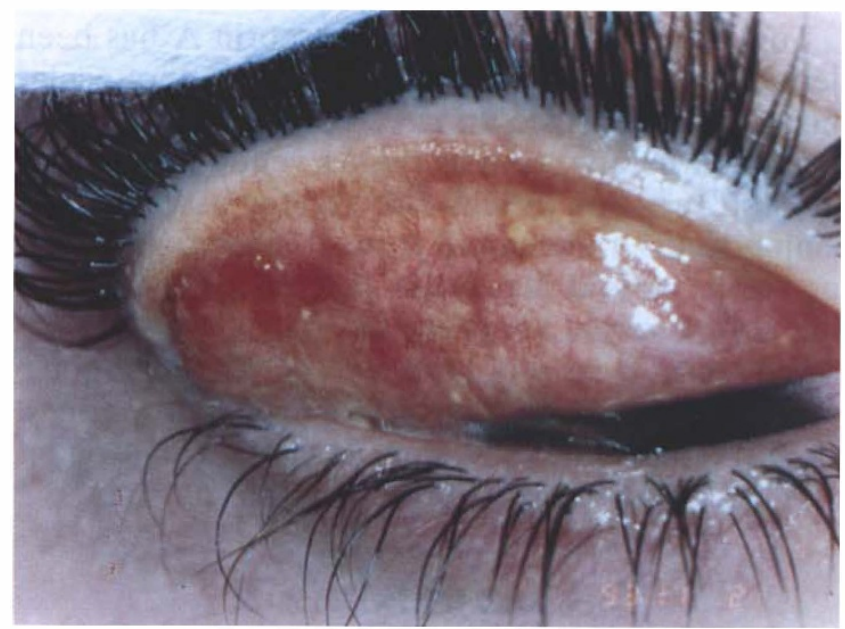

(c)

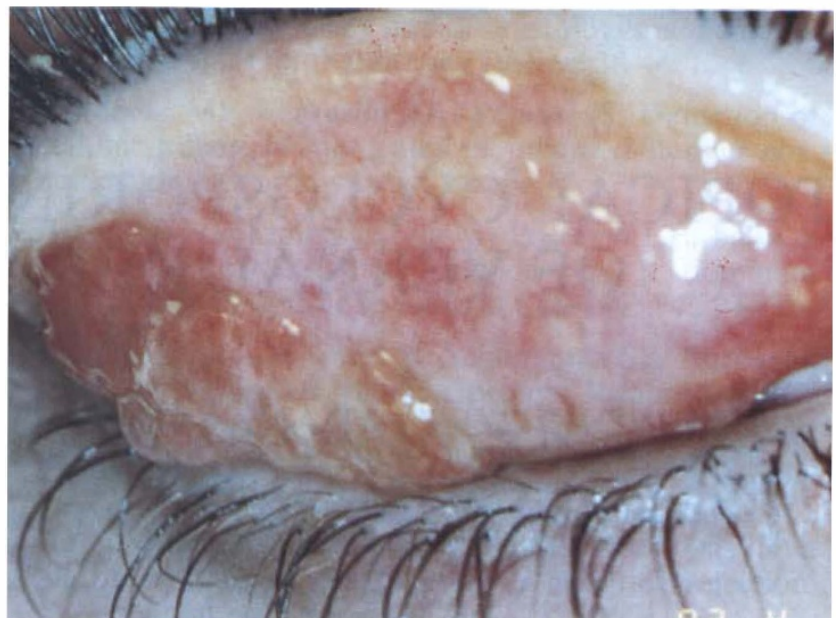

(b)

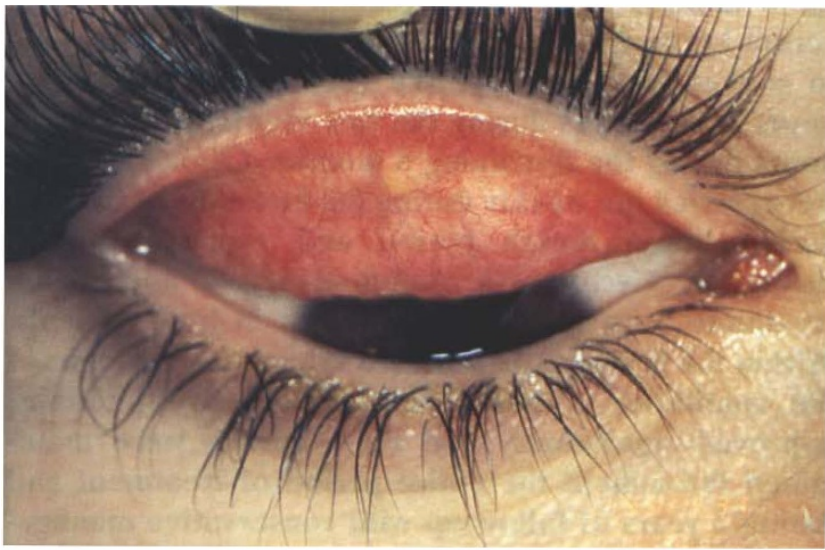

(d)

Fig. 1. Case 2. (a) Giant papillary proliferations of right upper eyelid. (b) After 1 month of cyclosporin A $2 \%$ eyedrops. (c) After 3 months of treatment. (d) After 2 years of follow-up without treatment.

\section{Treatment and Results}

Topical cyclosporin A $2 \%$ was prepared under sterile conditions using commercially available oral cyclosporin A diluted with olive oil. In both cases the treatment regimen was started with one drop of cyclosporin A four times daily for the first month and twice daily for the next 5 months. Both eyes were treated simultaneously. No additional therapy was administered during the treatment. Clinical ocular findings were recorded at entry, day 7 , day 15 , day 30 and then monthly. A month later, both patients showed improvement with relief of symptoms. Subsequent examination demonstrated a re-epithelisation of the cornea, and a decrease in congestion of conjunctival vessels and in perilimbalinjection. After the first month both patients indicated a marked improvement of their symptoms and the slit lamp examination showed a total stabilisation of the corneal epithelium associated with a pronounced decrease in papillary proliferations (Figs. 1, 2). Intraocular pressure was not affected. Visual acuity improved because of a substantial decrease in photophobia. Both patients benefited from the treatment with cyclosporin by remaining free of the disease during the other 5 months of treatment. After cessation of the cyclosporin, no recurrence of vernal symptoms was recorded during over 2 years of follow-up with conservative management.

The side effects of cyclosporin $2 \%$ eyedrops were minimal. Radio-immunoassay with polyclonal antibodies detected no cyclosporin blood levels. Kidney and liver function tests showed no significant change before or after treatment in either patient. Neither of the patients developed ocular surface toxicity of any significance; only a mild conjunctival hyperaemia manifested itself during the first week of treatment. 


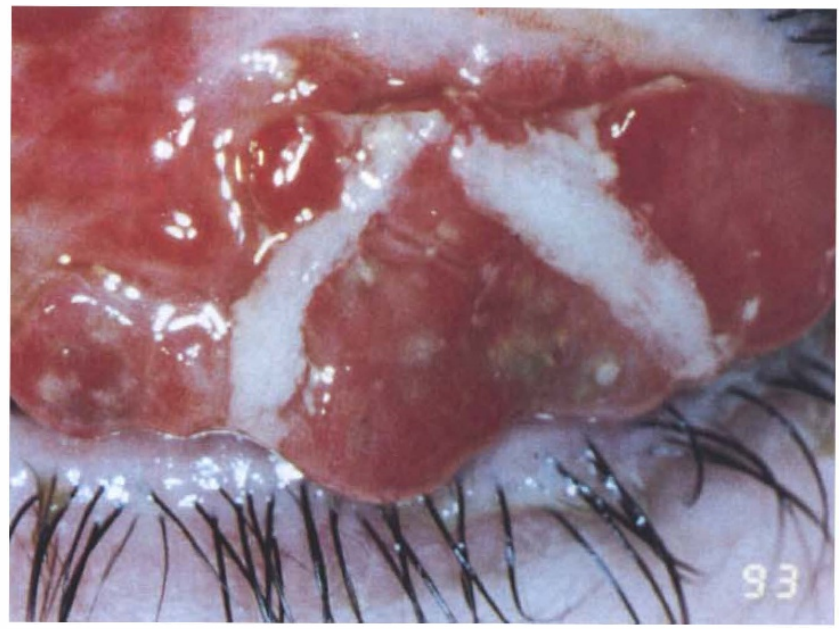

(a)

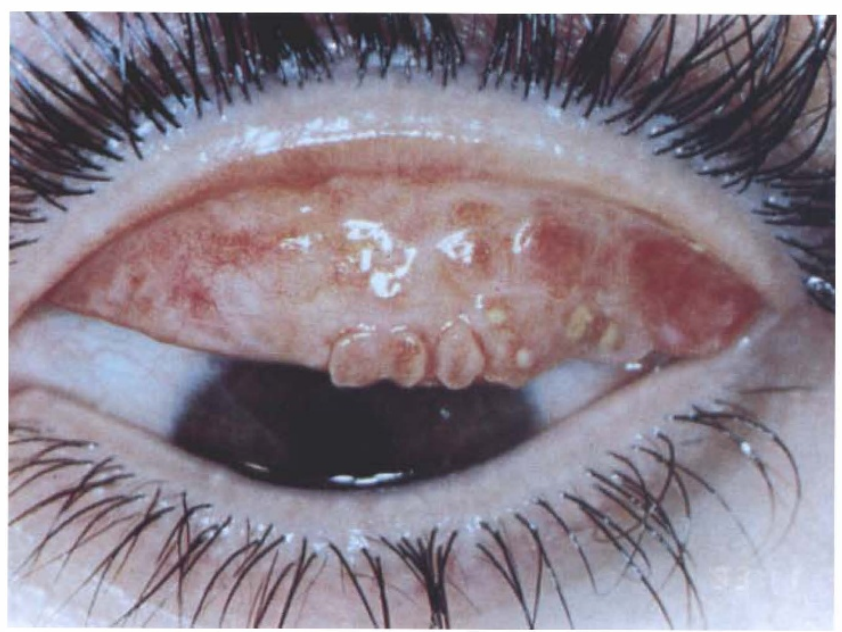

(c)

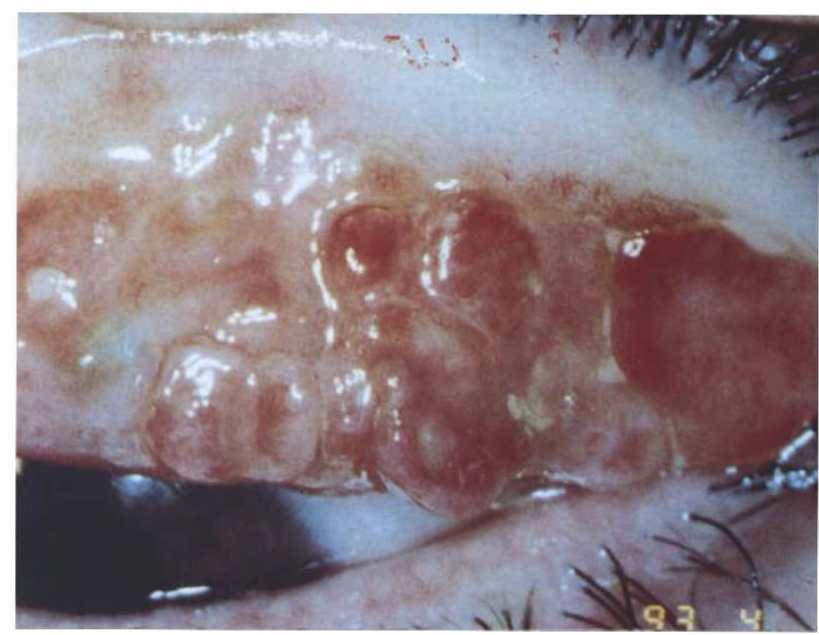

(b)

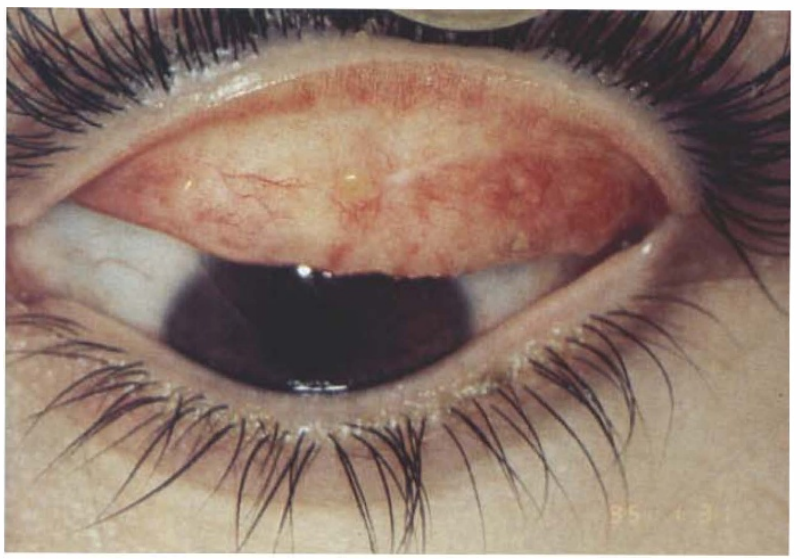

(d)

Fig. 2. Case 2. (a) Giant papillary proliferations of left upper eyelid. (b) After 1 month of cyclosporin A $2 \%$ eyedrops. (c) After 3 months of treatment. (d) After 2 years of follow-up without treatment.

\section{DISCUSSION}

Vernal keratoconjunctivitis is a common disorder worldwide that in most cases can be effectively managed by conservative measures. However, severe cases may lead to significant distress in patients, who may be unable to participate in normal daily activities because of intense symptoms such as itching, photophobia, tear-shedding, pain and mucous discharge. In these refractory situations, vision loss occurs because of corneal complications of the disease or because of the abuse of corticosteroids, which may lead to cataracts, glaucoma and secondary infections. Because of the potential sequelae of systemic corticosteroids in children and the rapid recurrence of symptoms following their discontinuation, some clinical trials have been started with topical cyclosporin, which has been demonstrated to be effective in controlling both conjunctival tarsal and limbal forms of vernal keratoconjunctivitis. ${ }^{1-6}$
Cyclosporin A is a potent immunomodulator which is highly effective in the control of cellmediated immunopathological conditions but is also believed to play a role in the control of immediate hypersensitivity reactions by means of an inhibition in cooperation between $\mathrm{T}$ and $\mathrm{B}$ cells; cyclosporin may thus influence the production of $\mathrm{IgE}$ by $\mathrm{B}$ cells by its influence on the $\mathrm{T}$ helper population. ${ }^{1,2,4-6}$ Some studies have shown that active vernal keratoconjunctivitis produces an increase in T-lymphocytedependent mast cells in the epithelial cells of conjunctival biopsies. ${ }^{4}$ These findings permit us to suppose that conjunctival basophil hypersensitivity might play a role in the pathogenesis of the illness and that this phenomenon could be suppressed by cyclosporin.

Oral cyclosporin A is associated with significant systemic side effects such as gingival hyperplasia, hypertrichosis, anaemia, hepatotoxicity, hypertension and nephrotoxicity, so that systemic use of the drug in anterior segment inflammatory disease has 
been limited and topical administration has been evaluated. Cyclosporin $\mathrm{A}$ is a neutral hydrophobic peptide that can easily penetrate the corneal epithelium. Topical cyclosporin eyedrops allow significant levels of the drug in the epithelium, stroma, sclera and conjunctiva, so it seems appropriate to consider its use in the treatment of immunologically mediated corneal and conjunctival diseases.

We found a dramatic improvement in symptoms and signs in both our patients with severe vernal keratoconjunctivitis. Both patients showed a marked decrease in 'rough cobblestones' after 4 weeks of treatment. These results differ from most of the cases previously reported where papillary hyperplasia revealed little change after treatment. ${ }^{1-5}$

We consider that topical cyclosporin A represents an important addition to the therapeutic armamentarium because it is effective in controlling the symptoms and signs of vernal keratoconjunctivitis and seems to be well tolerated both locally and systemically.
Key words: Cyclosporin A, Vernal keratoconjunctivitis, Ocular inflammatory disease.

\section{REFERENCES}

1. BenEzra D, Pe'er J, Brodsky M, Cohen E. Cyclosporine eyedrops for the treatment of severe vernal keratoconjunctivitis. Am J Ophthalmol 1986;101:278.

2. BenEzra D, Matamoros N, Cohen E. Treatment of severe vernal karatoconjunctivitis with cyclosporine A eyedrops. Transplant Proc 1988;22(2)(Suppl 2):644-9.

3. Secchi A, Tognon S, Leonardi A. Topical use of cyclosporine in the treatment of vernal keratoconjunctivitis. Am J Ophthalmol 1990;110:641-5.

4. Bleik J, Tabbara K. Topical cyclosporine in vernal keratoconjunctivitis. Ophthalmology 1991;98:1679-84.

5. Holland E, Olsen T, Ketcham J, Florine C, Krachmer J, Purcell J, et al. Topical cyclosporin A in the treatment of anterior segment inflammatory disease. Cornea 1993; 12:413-9.

6. Kaan G, Özden Ö. Therapeutic use of topical cyclosporine. Ann Ophthalmol 1993;25:182-6. 